International Journal of Pure and Applied Mathematics

Volume 90 No. 2 2014, 109-117

ISSN: 1311-8080 (printed version); ISSN: 1314-3395 (on-line version)

url: http://www.ijpam.eu

doi: http://dx.doi.org/10.12732/ijpam.v90i2.1

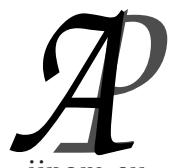

ijpam.eu

\title{
AN APPLICATION OF BINOMIAL COEFFICIENTS TO SOCIAL CHOICE THEORY
}

\author{
Bonifacio Llamazares \\ Department of Applied Economics and Institute of Mathematics (IMUVA) \\ University of Valladolid \\ Avda. Valle de Esgueva 6, 47011, Valladolid, SPAIN
}

\begin{abstract}
In this paper we determine the number of positive integer sequences $a_{1}, a_{2}, \ldots, a_{k}$ such that $1 \leq a_{1} \leq \cdots \leq a_{k} \leq m$ and $a_{i} \geq i$ for all $i \in\{1, \ldots, k\}$. After that, we apply this result to calculate the number of anonymous, neutral and monotonic social welfare functions when only two alternatives are considered.
\end{abstract}

AMS Subject Classification: 11B65, 91B12, $91 \mathrm{~B} 14$

Key Words: binomial coefficients, voting systems, social welfare functions

\section{Introduction}

Binomial coefficients are closely related to counting problems and they appear in many scientific fields. As is well known, the binomial coefficient $\left(\begin{array}{c}m \\ k\end{array}\right)$ is the number of $k$-element subsets of $\{1, \ldots, m\}$. When the repetition of elements is allowed, we find the multichoose coefficient, $\left.\left(\begin{array}{c}m \\ k\end{array}\right)\right)$, defined by

$$
\left(\left(\begin{array}{c}
m \\
k
\end{array}\right)\right)=\left(\begin{array}{c}
m+k-1 \\
k
\end{array}\right) \text {. }
$$

An equivalent interpretation is that $\left.\left(\begin{array}{c}m \\ k\end{array}\right)\right)$ is the number of positive integer sequences $a_{1}, a_{2}, \ldots, a_{k}$ such that $1 \leq a_{1} \leq \cdots \leq a_{k} \leq m$ (see, for instance, Benjamin and Quinn [1]).

Received: May 25, 20012

(c) 2014 Academic Publications, Ltd. url: www.acadpubl.eu 
In this paper we focus on the previous sequences whose elements satisfy an additional condition, $a_{i} \geq i$ for all $i \in\{1, \ldots, k\}$, and we calculate their number. Next, this number will be applied to the field of Social Choice by determining the number of social welfare functions (SWFs) that satisfy anonymity, neutrality and monotonicity.

The paper is organized in two sections. The first section provides the main result of this paper. In the second section we apply this result to Social Choice Theory.

\section{The Result}

Before providing the result, we recall the definition and some properties of binomial coefficients (see, for instance, Riordan [7] and Benjamin and Quinn [1]).

Remark 1. Let $m \in \mathbb{N}$ and $k \in \mathbb{Z}$. Then:

1. $\left(\begin{array}{c}m \\ k\end{array}\right)= \begin{cases}\frac{m !}{k !(m-k) !}, & \text { if } 0 \leq k \leq m, \\ 0, & \text { otherwise. }\end{cases}$

2. $\left(\begin{array}{c}m \\ k\end{array}\right)=\left(\begin{array}{c}m \\ m-k\end{array}\right)$.

3. $\left(\begin{array}{c}m \\ k\end{array}\right)+\left(\begin{array}{c}m \\ k+1\end{array}\right)=\left(\begin{array}{c}m+1 \\ k+1\end{array}\right)$.

4. If $k \geq 0$, then $\sum_{i=k}^{m}\left(\begin{array}{l}i \\ k\end{array}\right)=\left(\begin{array}{l}m+1 \\ k+1\end{array}\right)$.

Now we determine the number of positive integer sequences $a_{1}, a_{2}, \ldots, a_{k}$ such that $1 \leq a_{1} \leq \cdots \leq a_{k} \leq m$ and $a_{i} \geq i$ for all $i \in\{1, \ldots, k\}$. For any set $S$, \# $S$ will denote the cardinal of $S$.

Proposition 2. Given $k, m \in \mathbb{N}$ with $k \geq 1$ and $m \geq k$, let $s_{k}^{m}=\# S_{k}^{m}$, where

$$
S_{k}^{m}=\left\{\left(a_{1}, \ldots, a_{k}\right) \in \mathbb{N}^{k} \mid \begin{array}{l}
1 \leq a_{1} \leq \cdots \leq a_{k} \leq m, \\
a_{i} \geq i, i=1, \ldots, k
\end{array}\right\} .
$$

Then

$$
s_{k}^{m}=\left(\begin{array}{c}
m+k-1 \\
k
\end{array}\right)-\left(\begin{array}{c}
m+k-1 \\
k-2
\end{array}\right) .
$$


Proof. The proof is by induction on $k$. If $k=1$, then

$$
\begin{aligned}
s_{1}^{m} & =\#\left\{a_{1} \in \mathbb{N} \mid 1 \leq a_{1} \leq m\right\}=m \\
& =\left(\begin{array}{c}
m+1-1 \\
1
\end{array}\right)-\left(\begin{array}{c}
m+1-1 \\
-1
\end{array}\right),
\end{aligned}
$$

where the last equality is due to (1) of Remark 1. Suppose now the result is true for $k=p$, where $p \leq m-1$, and let's see that the claim holds for $k=p+1$. Since $m \geq p+1, s_{p+1}^{m}$ can be calculated taking into account that the largest element of the sequence, $a_{p+1}$, ranges between $p+1$ and $m$, that is,

$$
\begin{aligned}
S_{p+1}^{m} & =\left\{\left(a_{1}, \ldots, a_{p+1}\right) \in \mathbb{N}^{p+1} \mid \begin{array}{l}
1 \leq a_{1} \leq \ldots \leq a_{p+1} \leq m, \\
a_{i} \geq i, i=1, \ldots, p+1
\end{array}\right\} \\
& =\bigcup_{j=p+1}^{m}\left\{\left(a_{1}, \ldots, a_{p}, j\right) \in \mathbb{N}^{p+1}, \begin{array}{l}
1 \leq a_{1} \leq \ldots \leq a_{p} \leq j \\
a_{i} \geq i, i=1, \ldots, p
\end{array}\right\} .
\end{aligned}
$$

Therefore, taking into account (4) and (2) of Remark 1, we have

$$
\begin{aligned}
s_{p+1}^{m} & =\sum_{j=p+1}^{m} s_{p}^{j}=\sum_{j=p+1}^{m}\left[\left(\begin{array}{c}
j+p-1 \\
p
\end{array}\right)-\left(\begin{array}{c}
j+p-1 \\
p-2
\end{array}\right)\right] \\
& =\sum_{i=2 p}^{m+p-1}\left(\begin{array}{l}
i \\
p
\end{array}\right)-\sum_{i=2 p}^{m+p-1}\left(\begin{array}{c}
i \\
p-2
\end{array}\right)=\left(\sum_{i=p}^{m+p-1}\left(\begin{array}{l}
i \\
p
\end{array}\right)-\sum_{i=p}^{2 p-1}\left(\begin{array}{l}
i \\
p
\end{array}\right)\right) \\
& -\left(\sum_{i=p-2}^{m+p-1}\left(\begin{array}{c}
i \\
p-2
\end{array}\right)-\sum_{i=p-2}^{2 p-1}\left(\begin{array}{c}
i \\
p-2
\end{array}\right)\right) \\
& =\left(\left(\begin{array}{c}
m+p \\
p+1
\end{array}\right)-\left(\begin{array}{c}
2 p \\
p+1
\end{array}\right)\right)-\left(\left(\begin{array}{c}
m+p \\
p-1
\end{array}\right)-\left(\begin{array}{c}
2 p \\
p-1
\end{array}\right)\right) \\
& =\left(\begin{array}{c}
m+p \\
p+1
\end{array}\right)-\left(\begin{array}{c}
m+p \\
p-1
\end{array}\right) .
\end{aligned}
$$

\section{An Application to Social Choice Theory}

One of the most important issues in the field of Social Choice is the analysis of social welfare functions (SWFs), that is, procedures for determining a collective 
preference from the opinions of a group of $n$ individuals on a set of alternatives. The simplest case is when only two alternatives are considered. However, this case is not trivial and it has generated a vast literature on the subject. The aim of this section is to determine, in this case, the number of SWFs that satisfy the following properties: anonymity, neutrality and monotonicity. For this, we will apply the result obtained in the previous section.

Next we introduce basic concepts and notation on SWFs. Let $N=\{1, \ldots, n\}$ be the set of voters, with $n \geq 2$, and $x, y$ two alternatives. The individual preferences between both alternatives are described by a profile $D=\left(d_{1}, \ldots, d_{n}\right)$, where $d_{i}$ is $1,-1$ or 0 depending on whether individual $i$ prefers $x$ to $y, y$ to $x$ or is indifferent between both alternatives. The set of profiles of preferences will be denoted by $\mathcal{D}$.

Given $D, D^{\prime} \in \mathcal{D}$ and $\sigma$ a permutation of $N$, we will use the following notation: $n^{+}(D)=\#\left\{i \in N \mid d_{i}=1\right\}, n^{-}(D)=\#\left\{i \in N \mid d_{i}=-1\right\}$, $-D=\left(-d_{1}, \ldots,-d_{n}\right), D_{\sigma}=\left(d_{\sigma(1)}, \ldots, d_{\sigma(n)}\right)$ and $D^{\prime} \geq D$ will mean $d_{i}^{\prime} \geq d_{i}$ for all $i \in N$. Moreover, given $a \in \mathbb{R},\lfloor a\rfloor$ will denote the integer part of $a$, i.e., the largest integer smaller than or equal to $a$.

For each profile of preferences, the collective preference will be obtained by means of SWFs.

Definition 3. A SWF is a mapping $F: \mathcal{D} \longrightarrow\{-1,0,1\}$.

The possible values of $F, 1,0$, and -1 , have a similar interpretation as that in the case of individual preferences.

Next we present some well-known properties of SWFs: Anonymity, neutrality and monotonicity. The first one, anonymity, guarantees an equalitarian treatment for all individuals. Similarly, neutrality assures that both alternatives are treated equally. Finally, monotonicity means that increased support for an alternative does not hurt this alternative.

Definition 4. Let $F$ be a SWF.

1. $F$ is anonymous if for all permutation $\sigma$ of $N$ and all profile $D \in \mathcal{D}$ we have $F\left(D_{\sigma}\right)=F(D)$.

2. $F$ is neutral if for all profile $D \in \mathcal{D}$ we have $F(-D)=-F(D)$.

3. $F$ is monotonic if for all pair of profile $D, D^{\prime} \in \mathcal{D}$ such that $D^{\prime} \geq D$ we have $F\left(D^{\prime}\right) \geq F(D)$.

It is worth noting that if $F$ is anonymous, then the value $F(D)$ depends on only of $n^{+}(D)$ and $n^{-}(D)$. On the other hand, any neutral SWF is characterized 
by the set $F^{-1}(\{1\})$, since

$$
\begin{aligned}
F^{-1}(\{-1\}) & =\left\{D \in \mathcal{D} \mid-D \in F^{-1}(\{1\})\right\}, \\
F^{-1}(\{0\}) & =\mathcal{D} \backslash\left(F^{-1}(\{1\}) \cup F^{-1}(\{-1\})\right) .
\end{aligned}
$$

It is easy to check that there exist $3^{3^{n}}$ different SWFs. As new properties are imposed on the SWFs, the number of them is diminished. For instance, Perry and Powers $[5,6]$ have calculated the number of SWFs that satisfy anonymity, $3^{\left(n^{2}+3 n+2\right) / 2}$, anonymity and neutrality, $3^{\left\lfloor\left(n^{2}+2 n+1\right) / 4\right\rfloor}$, and anonymity and monotonicity, $\left(\begin{array}{c}2 n+3 \\ n+1\end{array}\right)$. On the other hand, Young et al. [8] have determined the number of SWFs that satisfy anonymity, neutrality and monotonicity, $\left(\begin{array}{c}n+1 \\ \left\lfloor\frac{n}{2}\right\rfloor+1\end{array}\right)$. It is worth noting that similar counting problems have also been considered by Freixas and Zwicker [4] and Campbell et al. [2].

In the sequel we get the same number as obtained by Young et al. [8], although by a different approach. From a result given by Fishburn [3, p. 56], we reduce SWFs to positive integer sequences that express the number of votes needed for an alternative to win, according to the number of votes obtained by the other alternative. However, Young et al. [8] specify these sequences according to the number of tie votes. For this reason, these sequences are quite different and the procedure for obtaining the number of anonymous, neutral and monotonic SWFs is also completely different.

We begin with the result given by Fishburn [3, p. 56], which can be given as a characterization of anonymous, neutral and monotonic SWFs.

Theorem 5. Let $F$ be a $S W F$. Then the following statements are equivalent:

1. $F$ is anonymous, neutral and monotonic.

2. There exist integers $r_{0} \leq r_{1} \leq \cdots \leq r_{\left\lfloor\frac{n-1}{2}\right\rfloor}$ that satisfy $r_{i} \geq i$ for all $i \in\left\{0,1, \ldots,\left\lfloor\frac{n-1}{2}\right\rfloor\right\}$ and such that

$$
F(D)=\left\{\begin{aligned}
1, & \text { if } n^{+}(D)>r_{n^{-}(D)} \\
-1, & \text { if } n^{-}(D)>r_{n^{+}(D)} \\
0, & \text { otherwise. }
\end{aligned}\right.
$$

Let $\mathcal{R}$ be the set of vectors $\left(r_{0}, r_{1}, \ldots, r_{\left\lfloor\frac{\mathrm{n}-1}{2}\right\rfloor}\right)$ such that the integers $r_{i}$ satisfy the conditions given in the previous theorem. According to this theorem, given $\mathbf{r} \in \mathcal{R}$, we can associate with $\mathbf{r}$ an anonymous, neutral and monotonic SWF, $F_{\mathbf{r}}$. 
The number of anonymous, neutral and monotonic SWFs is going to be obtained through the vectors of $\mathcal{R}$. However, given that different vectors $\mathbf{r}, \mathbf{r}^{\prime} \in$ $\mathcal{R}$ can generate the same SWF, we firstly need to characterize these vectors. For this, given $\mathbf{r} \in \mathcal{R}$, we will use the following notation:

$$
N_{\mathbf{r}}=\left\{i \in\left\{0,1, \ldots,\left\lfloor\frac{n-1}{2}\right\rfloor\right\} \quad \mid i+r_{i} \leq n-1\right\} .
$$

The set $N_{\mathbf{r}}$ is closely connected with the SWF $F_{\mathbf{r}}$, as we show in the following lemma.

Lemma 6. Let $\mathbf{r} \in \mathcal{R}$. Then the following statements are equivalent:

1. $i \in N_{\mathbf{r}}$.

2. There exists $D \in \mathcal{D}$ such that $n^{-}(D)=i$ and $F_{\mathbf{r}}(D)=1$.

Proof.

$(1) \Rightarrow(2)$ : Given $i \in N_{\mathbf{r}}$, let $D \in \mathcal{D}$ a profile such that $n^{-}(D)=i$ and $n^{+}(D)=r_{i}+1$. This profile exists because $n^{-}(D)+n^{+}(D)=i+r_{i}+1 \leq n$. Moreover, $F_{\mathbf{r}}(D)=1$ since $n^{+}(D)>r_{n^{-}(D)}$.

$(2) \Rightarrow(1)$ : Suppose that there exists $D \in \mathcal{D}$ such that $n^{-}(D)=i$ and $F_{\mathbf{r}}(D)=1$. Since $F_{\mathbf{r}}(D)=1$, we have $n^{+}(D)>r_{n^{-}(D)}=r_{i}$. Therefore, $i+r_{i}<n^{-}(D)+n^{+}(D) \leq n$ and, consequently, $i \in N_{\mathrm{r}}$.

Now, we are going to characterize the vectors that generate the same SWFs.

Proposition 7. Let $\mathbf{r}, \mathbf{r}^{\prime} \in \mathcal{R}$. Then the following statements are equivalent:

1. $F_{\mathrm{r}}=F_{\mathrm{r}^{\prime}}$.

2. $N_{\mathbf{r}}=N_{\mathbf{r}^{\prime}}$ and $r_{i}=r_{i}^{\prime}$ for all $i \in N_{\mathbf{r}}$.

Proof.

$(1) \Rightarrow(2)$ : First, we are going to prove that $N_{\mathbf{r}}=N_{\mathbf{r}^{\prime}}$. By Lemma $6, i \in N_{\mathbf{r}}$ if and only if there exists $D \in \mathcal{D}$ such that $n^{-}(D)=i$ and $F_{\mathbf{r}}(D)=1$. Since $F_{\mathbf{r}}=F_{\mathbf{r}^{\prime}}$, then there exists $D \in \mathcal{D}$ such that $n^{-}(D)=i$ and $F_{\mathbf{r}^{\prime}}(D)=1$ and, again by Lemma 6 , this is equivalent to $i \in N_{\mathbf{r}^{\prime}}$.

Now, we are going to prove that $r_{i}=r_{i}^{\prime}$ for all $i \in N_{\mathbf{r}}$. This is proven by contradiction. Suppose that there exists $i \in N_{\mathbf{r}}$ such that $r_{i} \neq r_{i}^{\prime}$. We can suppose, without loss of generality, that $r_{i}<r_{i}^{\prime}$. Consider a profile $D \in \mathcal{D}$ such that $n^{-}(D)=i$ and $n^{+}(D)=r_{i}+1$ (this profile exists because $n^{-}(D)+n^{+}(D)=$ $\left.i+r_{i}+1 \leq n\right)$. For this profile we have $F_{\mathbf{r}}(D)=1$ and $F_{\mathbf{r}^{\prime}}(D)<1$; i.e., a contradiction. 
$(2) \Rightarrow(1)$ : Since $F_{\mathbf{r}}$ and $F_{\mathbf{r}^{\prime}}$ are neutral, it is sufficient to prove that $F_{\mathbf{r}}(D)=$ 1 if and only if $F_{\mathbf{r}^{\prime}}(D)=1$ for all $D \in \mathcal{D}$. Let $D \in \mathcal{D}$ such that $F_{\mathbf{r}}(D)=1$. By Lemma $6, n^{-}(D) \in N_{\mathbf{r}}$, and

$$
F_{\mathbf{r}}(D)=1 \Rightarrow n^{+}(D)>r_{n^{-}(D)} \Rightarrow n^{+}(D)>r_{n^{-}(D)}^{\prime} \Rightarrow F_{\mathbf{r}^{\prime}}(D)=1 .
$$

In a similar way we can prove that $F_{\mathbf{r}^{\prime}}(D)=1 \Rightarrow F_{\mathbf{r}}(D)=1$ for all $D \in \mathcal{D}$.

The previous proposition allows us to determine, in a methodical way, the SWFs that satisfy anonymity, neutrality and monotonicity. Let's see an example.

Example 8. Consider the case $n=3$. For this value,

$$
\mathcal{R}=\left\{\left(r_{0}, r_{1}\right) \in \mathbb{N}^{2} \mid 0 \leq r_{0} \leq r_{1}, r_{1} \geq 1\right\},
$$

and given $\mathbf{r} \in \mathcal{R}, N_{\mathbf{r}}=\left\{i \in\{0,1\} \mid r_{i} \leq 2-i\right\}$. Therefore:

1. If $N_{\mathbf{r}}=\emptyset$, then we have the null SWF: $F_{\mathbf{r}}(D)=0$ for all $D \in \mathcal{D}$.

2. If $N_{\mathrm{r}}=\{0\}$, then $r_{0}$ can take any value between 0 and 2 . So,

(a) If $r_{0}=0$, then we obtain Pareto majority:

$$
F_{\mathbf{r}}(D)=1 \Leftrightarrow n^{+}(D)>0 \text { and } n^{-}(D)=0 .
$$

(b) If $r_{0}=1$, then we have the SWFs defined by:

$$
F_{\mathbf{r}}(D)=1 \Leftrightarrow n^{+}(D)>1 \text { and } n^{-}(D)=0 .
$$

(c) If $r_{0}=2$, then we obtain unanimous majority:

$$
F_{\mathbf{r}}(D)=1 \Leftrightarrow n^{+}(D)=3 .
$$

3. If $N_{\mathbf{r}}=\{0,1\}$, then $r_{1}=1$ and $0 \leq r_{0} \leq r_{1}$. Thus,

(a) If $\left(r_{0}, r_{1}\right)=(0,1)$, then we have simple majority:

$$
F_{\mathbf{r}}(D)=1 \Leftrightarrow n^{+}(D)>n^{-}(D) .
$$

(b) If $\left(r_{0}, r_{1}\right)=(1,1)$, then we obtain absolute majority:

$$
F_{\mathbf{r}}(D)=1 \Leftrightarrow n^{+}(D)>1 .
$$


According to Proposition 7, the number of anonymous, neutral an monotonic SWFs, $S_{n}$, can be determined through the following expression:

$$
S_{n}=1+\sum_{j=1}^{\left\lfloor\frac{n+1}{2}\right\rfloor} \# N_{j}^{n-j}
$$

where $N_{j}^{n-j}, j=1, \ldots,\left\lfloor\frac{n+1}{2}\right\rfloor$, are the sets defined by

$$
N_{j}^{n-j}=\left\{\begin{array}{ll}
\left(r_{0}, \ldots, r_{j-1}\right) \in \mathbb{N}^{j} \mid \begin{array}{l}
0 \leq r_{0} \leq \cdots \leq r_{j-1} \leq n-j \\
r_{i} \geq i, i=0, \ldots, j-1
\end{array}
\end{array}\right\} .
$$

Consider now the function $h: N_{j}^{n-j} \longrightarrow S_{j}^{n+1-j}$ defined by

$$
h\left(r_{0}, \ldots, r_{j-1}\right)=\left(r_{0}+1, \ldots, r_{j-1}+1\right) .
$$

It is easy to check that $h$ is a biyection. Therefore, by Proposition 2, we have $\# N_{j}^{n-j}=\# S_{j}^{n+1-j}=s_{j}^{n+1-j}=\left(\begin{array}{c}n \\ j\end{array}\right)-\left(\begin{array}{c}n \\ j-2\end{array}\right)$.

The previous remark allows us to get the number of SWFs that satisfy anonymity, neutrality and monotonicity.

Proposition 9. The number of anonymous, neutral and monotonic SWFS is $\left(\begin{array}{c}n+1 \\ \left\lfloor\frac{n+1}{2}\right\rfloor\end{array}\right)$.

Proof. According with (1) and (3) of Remark 1, we have:

$$
\begin{aligned}
S_{n} & =1+\sum_{j=1}^{\left\lfloor\frac{\mathrm{n}+1}{2}\right\rfloor} s_{j}^{n+1-j}=\left(\begin{array}{c}
n \\
0
\end{array}\right)+\sum_{j=1}^{\left\lfloor\frac{\mathrm{n}+1}{2}\right\rfloor}\left[\left(\begin{array}{c}
n \\
j
\end{array}\right)-\left(\begin{array}{c}
n \\
j-2
\end{array}\right)\right] \\
& =\sum_{j=0}^{\left\lfloor\frac{\mathrm{n}+1}{2}\right\rfloor}\left(\begin{array}{c}
n \\
j
\end{array}\right)-\sum_{j=0}^{\left\lfloor\frac{n+1}{2}\right\rfloor-2}\left(\begin{array}{l}
n \\
j
\end{array}\right)=\left(\begin{array}{c}
n \\
\left\lfloor\frac{n+1}{2}\right\rfloor-1
\end{array}\right)+\left(\begin{array}{c}
n \\
\left\lfloor\frac{n+1}{2}\right\rfloor
\end{array}\right) \\
& =\left(\begin{array}{c}
n+1 \\
\left\lfloor\frac{n+1}{2}\right\rfloor
\end{array}\right) .
\end{aligned}
$$




\section{Acknowledgments}

This work is partially supported by the Spanish Ministry of Economy and Competitiveness (Project ECO2012-32178) and ERDF.

\section{References}

[1] A.T. Benjamin, J.J. Quinn, Proofs that Really Count: The Art of Combinatorial Proof, Mathematical Association of America, Dolciani Series, Washington DC (2003).

[2] D.E. Campbell, J. Graver, J.S. Kelly, There are more strategy-proof procedures than you think, Math. Soc. Sci., 64 (2012), 263-265.

[3] P.C. Fishburn, The Theory of Social Choice, Princeton University Press, Princeton (1973).

[4] J. Freixas, W.S. Zwicker, Anonymous yes-no voting with abstention and multiple levels of approval, Games Econ. Behav., 67 (2009), 428-444.

[5] J. Perry, R.C. Powers, Aggregation rules that satisfy anonymity and neutrality, Econ. Lett., 100 (2008), 108-110.

[6] J. Perry, R.C. Powers, Anonymity, monotonicity, and quota pair systems, Math. Soc. Sci., 60 (2010), 57-60.

[7] J. Riordan, Combinatorial Identities, John Wiley \& Sons, Inc., New York (1968).

[8] S.C. Young, A.D. Taylor, W.S. Zwicker, Counting quota systems: a combinatorial question from Social Choice Theory, Math. Mag., 68 (1995), $331-342$. 
